\title{
Sobre el Desinterés por la Historia de la Psicología Social
}

\section{On the Lack of Interest for the History of Social Psychology}

\author{
Jaume Masip* \\ Universidad de Salamanca, España
}

(Recepción: Octubre 2007 - Aceptación: Noviembre 2007)

\begin{abstract}
Resumen
El conocimiento del pasado de una disciplina es imprescindible para una comprensión adecuada de su presente. Sin embargo, los psicólogos sociales mainstream muestran cierto desinterés e ignorancia sobre el pasado de la psicología social. Esto puede deberse en parte al papel que juegan en su formación las historias “oficiales" de la disciplina. Así, los capítulos históricos del Handbook of Social Psychology abarcan un periodo histórico cada vez más limitado y se escriben desde una perspectiva presentista que toma el estado actual de la disciplina como el más desarrollado, considerando que el pasado ha ido conduciendo irremisiblemente a este avanzado presente. Esta concepción no refleja la verdadera evolución, en modo alguno lineal, de una disciplina. Sólo una historia contextualista puede hacerlo, y los jóvenes psicólogos sociales deben rastrear en textos contextualistas los desarrollos históricos de la psicología social.
\end{abstract}

Palabras clave: Psicología social, historia, presentismo.

\begin{abstract}
Knowing the past of a discipline is essential to fully understand its present. However, mainstream social psychologists show some lack of interest and poor knowledge about the past of social psychology. This might be a result of the role that "official" histories of the discipline play in their education. Indeed, the historical period covered by the relevant chapters of the Handbook of Social Psychology is being progressively shortened, and these chapters are written from a presentist perspective that takes the current state of the discipline as the most evolved of all, which is a natural consequence of its ever-improving past. This view does not reflect the actual development of a discipline, which is not linear. Only a contextualist history may reflect it, and the novice social psychologists must look at contextualist writings to understand the historical developments of social psychology.
\end{abstract}

Key words: Social psychology, history, presentism.

\footnotetext{
* Correspondencia a: Jaume Masip. Departamento de Psicología Social y Antropología, Universidad de Salamanca. Facultad de Psicología, Avda. de la Merced, 109-131, 37005 Salamanca, España. E-mail: jmasip@usal.es. Teléfono: 34923 294610.
} 


\section{Sobre el Desinterés por la Historia de la Psicología Social}

Es innegable que el conocimiento sobre la historia de una disciplina tiene una gran importancia. Por ello, el desinterés (y desconocomiento) de los jóvenes profesores noveles de psicología social sobre la historia de su disciplina no puede sino ser preocupante. Durante su formación doctoral (e incluso posdoctoral), los estudiantes se convierten en rigurosos investigadores. Pero esta sofisticación investigadora raras veces se acompaña de una meticulosa indagación en torno a los fundamentos de la psicología social. Muy al contrario, el nuevo especialista profundiza al máximo en su tema de investigación, olvidándose de otros ámbitos anejos -y relevantes- de su propia área de especialización; se adentra en los insondables caminos de los diseños experimentales, de las técnicas multivariadas de análisis de datos, etc., pero es ajeno a las bases epistemológicas sobre las cuales se asienta ese modo específico de estudiar la realidad, así como a los vericuetos históricos que han llevado a que la ciencia contemporánea se caracterice por este tipo concreto de aproximaciones.

Ciertamente, es sólo con el conocimiento y la comprensión del pasado que se adquiere una visión clara, así como lo bastante amplia, del presente (por ej., Blanco, 1993). Con esto se quiere decir no sólo que para entender la actual psicología social uno debe conocer su historia y su desarrollo (a esto alude el adjetivo "clara" de la frase anterior), sino también, sobre todo, que únicamente a través de dicho conocimiento histórico puede uno aprehender la verdadera amplitud de la psicología social, con su diversidad de teorías, paradigmas, métodos de investigación y objetos de estudio. Una amplitud que va mucho más allá de esa psicología social mayoritaria, de naturaleza individualista y centrada en el experimento de laboratorio como único método válido de investigación, que para muchos psicólogos sociales contemporáneos parece ser la única psicología social. Considerar, por ejemplo, que la psicología social es sólo psicológica, ignorando la vertiente sociológica de la misma, o reducir la psicología social a la psicología social experimental, no sólo es ignorar la plural realidad de la disciplina en nuestros días (por ej., House, 1977; Stryker, 1983, 1989; Weigert y Gecas, 1995), sino especialmente los orígenes históricos de la misma (más próximos a la sociología que a la psicología; véase, por ej., Collier, Minton y Reynolds, 1996) y su desarrollo, hasta hace relativamente poco mucho menos ligado al método experimental que en la actualidad (Danzinger, 2000; MacMartin y Winston, 2000). No significa ello que hoy la psicología social no sea mayoritariamente individualista y experimentalista; sólo se pretende apuntar que existen también otras corrientes que, si bien son minoritarias, pueden incluso encontrarse más cercanas a las raíces históricas de la disciplina, y que la "verdadera" psicología social no se limita a la que se viene haciendo en Occidente (fundamentalmente en los Estados Unidos) desde los tiempos de la II Guerra Mundial. Esto sólo puede aprehenderse con claridad a través de un minucioso estudio histórico. En palabras de Carl Graumann (1996):

En el presente, "psicología social" es el nombre para:

(1) una subdisciplina de la psicología, desde la cual se origina la mayor parte de la investigación y publicaciones sociopsicológicas;

(2) un campo de investigación en (micro)sociología;

(3) cualquiera de varias otras ciencias sociales; y

(4) toda la psicología social, "incluyendo sus dos subdivisiones principales y sus diversas áreas de especialización"...

Tanto la preeminencia de su variante psicológica como la diversidad de la psicología social en el contexto de las ciencias sociales se entienden mejor si uno presta atención a la historia de la disciplina (p. 301).

En su voluminosa introducción a la historia de la psicología, Hergenhahn (2000) enfatiza la utilidad de conocer la historia de la disciplina al escribir que "de la misma manera que al saber más sobre las experiencias pasadas de una persona entendemos mejor su conducta actual, obtenemos una mejor comprensión de la psicología actual al estudiar sus orígenes históricos" (p. 4). Ciertamente, como señala Hergenhahn, raras veces, si es que lo hacen alguna vez, las ideas aparecen en una disciplina de pronto y completamente formadas. Más bien se desarrollan a lo largo del tiempo, y "el poder ver las ideas en su perspectiva histórica permite al estudiante apreciar de forma más completa la temáti- 
ca de la psicología moderna" (p. 3). Estas palabras son extrapolables a la psicología social, como lo son a la mayoría de disciplinas científicas. El autor también señala que el conocimiento del pasado permite descubrir los orígenes históricos de ideas aparentemente modernas. Podríamos ilustrar esta apreciación de Hergenhahn con innumerables ejemplos de la psicología social. Por citar algunos, recordemos que el enfoque de las representaciones sociales de Serge Moscovici $(1961,1981)$ está explícitamente inspirado en las representaciones colectivas de Emile Durkheim (2004); la teoría de la desindividualización de Philip Zimbardo (1969; en español, véanse Huici, 1987, y Zimbardo, 1997) no puede sino traer a nuestra mente el pretérito trabajo de Gustave Le Bon (1895) sobre la psicología de las masas; y los mecanismos de desvinculación de moral propuestos por Albert Bandura (1990, 1999, 2004; en español, véanse Espinosa, 2001, y Garrido, Herrero y Masip, 2004) son en buena medida análogos a las técnicas de neutralización de los sociólogos Gresham Sykes y David Matza (1957).

En relación con esta "repetición histórica", Amalio Blanco (1993) denuncia la esterilidad intelectual que él percibe en la psicología social actual, reivindicando por ello la importancia del pasado para entender el presente de la disciplina:

[L]os clásicos resultan absolutamente imprescindibles para comprender lo que hay de sustantivo en una disciplina científica como la nuestra, tan escasa de desarrollos teóricos de largo alcance y tan propensa a réplicas empíricas teóricamente desnutridas (p. 16).

Pero la historia no sólo nos ayuda a entender ese presente baldío que vive de los logros del pasado al que se refiere Blanco. También las "nuevas" psicologías sociales que han ido emergiendo como respuesta a la crisis que sacudió a la disciplina durante los años 70 del pasado siglo, las cuales constituyen alternativas "rompedoras" respecto a la psicología social mayoritaria del presente, adquieren un nuevo sentido a la luz de la historia. Como señala Tomás Ibáñez (1990):

[L]a necesidad de encontrar y de escrutar minuciosamente los principales puntos de "bifurcación" en donde se habían decidido los caminos a seguir se hizo tanto más acuciante cuanto que algunas de las propuestas alternativas que surgían frente a la psicología social dominante ("mainstream") parecían entroncar precisamente con posibles líneas de desarrollo de la psicología social que se habían visto truncadas en diversos momentos del desarrollo de la disciplina (p. 17).

En este sentido pueden resultar de interés, entre muchas otras, las consideraciones sobre las raíces del constructivismo y las del construccionismo incluidas en dos escritos de Frederic Munné (1998, 1999), la profunda revisión histórica de José Luis Pinillos (2002) sobre el postmodernismo, o las observaciones de Lupicinio Íñiguez $(2000,2003)$ de que algunos psicólogos sociales "fundacionales" en la psicología social mayoritaria (Asch, Heider y Sherif), así como los contenidos de algunos manuales de los sesenta (Brown, 1965; Shibutani, 1961), se encontraban sorprendentemente próximos a algunas de las propuestas de las recientes psicologías sociales críticas.

Por su parte, Graumann (1993) señala varias funciones de una historia disciplinar. En primer lugar, una función de identidad. Es decir, la historia permite a una disciplina como la psicología social, ciertamente muy heterogénea en cuanto al objeto de estudio, métodos, teorías, corrientes de investigación y "parentescos" disciplinares (con la sociología, con la psicología, con la antropología...) "definir y delimitar nuestra contribución al desarrollo del saber frente a otras disciplinas que pueden abordar temas o problemáticas similares" (Sabucedo, D’Adamo y García, 1997, p. 1). En relación con esta función de identidad es interesante notar que Graumann (1993; también Hewstone y Manstead, 1996) señala que los psicólogos sociales adscritos a la sociología y aquellos adscritos a la psicología tienen diferentes historias, con diferentes "pioneros" y "héroes". Esto permite no sólo constatar el pluralismo de la actual psicología social (véase, por ej., Munné, 1986, 1991, 1993), sino también advertir que sin una adecuada y completa visión histórica no se podrá aprehender la totalidad del panorama actual de nuestro campo.

La segunda función de la historia de una disciplina que señala Graumann (1993) es la función justificadora: "relacionándonos historiográficamente a nosotros mismos y a nuestra investigación 
actual con los logros 'clásicos', con las reputadas teorías del pasado o con los 'grandes hombres' justificamos nuestro propio trabajo y posiblemente incrementamos nuestro prestigio científico" (Graumann, 1993, p. 22). Esta función adquiere importancia capital en las historias llamadas "presentistas" (Stocking, 1965), que, frente a perspectivas historiográficas de naturaleza más historicista y contextualista, reconstruyen el pasado a partir del presente, adoptando una postura justificacionista y glorificadora del momento actual, aun a riesgo de alterar lo acontecido para adaptarlo a su "natural" culminación en el estado actual de las cosas. Este tipo de perspectiva corre el riesgo de desterrar en el olvido a notables contribuciones de nuestros predecesores, así como de sobrevalorar la importancia histórica de contribuciones sólo circunstancialmente relacionadas con el presente. Volveremos al tema del presentismo historiográfico en breve.

Graumann (1993) también señala que es posible aprender de la historia, siempre y cuando ésta tenga "en consideración las discontinuidades, inconvenientes, fracasos y callejones sin salida, así como las continuidades, éxitos y progresos. No debe pretender unidad si lo que hay es pluralismo, como ocurre en la psicología social” (p. 22). De nuevo critica Graumann la perspectiva presentista y justificacionista, y reivindica, como se hace en estas páginas, el valor de la historia para entender un presente diverso y plural. Como nos dice Hergenhahn (2000) al exponer las razones para estudiar la historia de la psicología, alguien con una conciencia histórica sabe de dónde procede la temática de su disciplina y las razones de que ésta se considere importante.

Queda clara, pues, la necesidad de todo psicólogo social de adentrarse en la historia de la disciplina. Sólo conociendo el pasado se puede entender el presente, un presente diverso y heterogéneo. El pasado ayuda además a conocer los contenidos de la psicología social y a trazar sus límites, a reconocer su importancia y a ubicarla en el mapa de las ciencias sociales, a construir la propia identidad, y a aprender de la historia evitando cometer de nuevo, como señala Hergenhahn (2000), los errores del pasado.

Dada esta innegable importancia que tiene el conocimiento de la historia de nuestra disciplina, cuesta comprender por qué, hasta hace relativamente pocos años, los psicólogos sociales se han mostrado tan ajenos a todo interés por la misma. Autores como Blanco (1993), Ibáñez (1990) y Jiménez Burillo (1987) han criticado con justicia dicho desinterés. El primero entresaca la siguiente cita de Sorokin (1957, en Blanco, 1993):

Los términos sociología moderna y ciencia psicosocial moderna se refieren al estado de estas disciplinas durante los veinte y cinco años pasados, aproximadamente. En este periodo el principal defecto de estas ciencias fue una especie de amnesia respecto a su historia, descubrimientos y realizaciones previas. Un segundo punto flaco está estrechamente ligado al primero. Muchos sociólogos o investigadores psicosociales modernos pretenden haber hecho determinado número de descubrimientos científicos por primera vez en la historia (p. 16).

Esta falta de memoria histórica disciplinar se hace patente al observar las escasas referencias antiguas que aparecen en los artículos publicados en las principales revistas de psicología social. Valga como anécdota ilustrativa cierta evaluación que recibió quien esto escribe de un trabajo empírico que había enviado a una revista para su publicación. Uno de los revisores señaló que algunas de las referencias eran demasiado antiguas. No quedó sino hacer un recuento de referencias por décadas -que puso tristemente de manifiesto que el revisor se equivocaba al estimar la frecuencia de textos antiguos citados- y mandárselo al editor junto con una apelación al indiscutible carácter de "clásicos” de la disciplina de las escasísimas referencias antiguas que sí se citaban en el texto.

Este olvido histórico resulta desconcertante cuando, por poner un caso, uno lee el texto de la charla que alguien de la indiscutible reputación en nuestro ámbito de estudio como es Ellen Berscheid dio en la sesión plenaria del congreso que celebraba el $25^{\circ}$ aniversario de la Society of Experimental Social Psychology (Berscheid, 1992). La impresión del lector es que, para Berscheid, la psicología social parece haber hecho acto de presencia en la escena de las ciencias sociales allá por los años 60 
del siglo XX, o a lo sumo poco antes. Escribe Berscheid: "Ahora, desde una distancia de 25 años y una fría mirada al contexto hostil en el que la psicología social se estaba desarrollando..." (Berscheid, 1992, p. 529, énfasis añadido). Al comentar la aparición de los textos de Brown (1965), Jones y Gerard (1967) y Sécord y Backman (1964), Berscheid (1992) afirma que tales textos constituían hitos de la psicología social "por varias razones, pero especialmente porque dieron forma y dirección al campo" (p. 529, énfasis añadido). La visión de Berscheid es la de que la psicología social era una ciencia débil, fragmentada y de escasa importancia en aquella época, y que fue a partir de entonces cuando adquirió una identidad propia y un empuje que la ha llevado hasta el "glorioso" presente.

Ibáñez (1990) señala como síntomas de ese desinterés por la historia la práctica inexistencia de trabajos sobre el tema con anterioridad a los años 60 del pasado siglo, así como la repetida publicación, en las sucesivas ediciones del Handbook of Social Psychology (Lindzey, 1954; Lindzey y Aronson, 1968, 1985), del mismo texto histórico de Gordon Allport (1954, 1968, 1985), reproducido una y otra vez prácticamente sin cambios durante nada menos que tres décadas ${ }^{1}$. Las razones que Ibáñez percibe tras ese olvido por la historia son lo que él entiende como la pretendida objetividad del método científico y la filosofía positivista en la que se instala la psicología social actual. Señala Ibáñez que, desde posturas positivistas, se considera que si los conocimientos de la psicología social son realmente científicos, entonces trascienden necesariamente las condiciones sociales e históricas en las que se han producido, y que si el científico cree firmemente que su labor consiste en descubrir ciertos principios objetivos, entonces no tendrá ningún interés en desentrañar los determinantes históricos, sociales e ideológicos que pudiera haber detrás de tales descubrimientos. Es necesario, según Ibáñez, que se resquebraje la "fe positivista" (sic) para poder advertir que las disciplinas científicas son el resultado de prácticas sociales propias de un momento histórico determinado y de una sociedad concreta. Al margen de lo acertado o no de su interpretación (probablemente parcial o incompleta) y en línea con la misma, Ibáñez (1990) observa que la crisis de la psicología social ha dejado como secuela un saludable interés por la historia de la disciplina, concretado en la aparición de revistas como el Journal for the Theory of Social Behavior (1971), la publicación de artículos sobre la historia en revistas mayoritarias como el American Psychologist (por ej., Danzinger, 1985; Pepitone, 1981), y la actividad de un nutrido grupo de autores que, desde distintos ámbitos geográficos, profundizan en el pasado de nuestra disciplina y reflexionan sobre el mismo (por ej., y aparte del propio Ibáñez en la obra citada, Erica Apfelbaum, Amalio Blanco, Kurt Danzinger, Robert Farr, Carl Graumann, John Greenwood, Florencio Jiménez Burillo, Ian Lubek, Frederic Munné, Franz Samelson, etc.).

Pese a este reciente interés, el grueso de los psicólogos sociales contemporáneos, principalmente los del otro lado del Atlántico y quienes se sitúan en las corrientes mayoritarias de la disciplina, siguen por lo común ignorantes del devenir histórico de la psicología social. A ello pueden haber contribuido las sucesivas ediciones del Handbook of Social Psychology. Como señalan Lubek y Apfelbaum (2000), las historias disciplinares "oficiales" como las de Gordon Allport (1954, 1968, 1985), Edward Jones (1985, 1998) y Shelley Taylor (1998) sirven a un doble propósito, retrospectivo y prospectivo. Por una parte, al especificar la naturaleza de la disciplina (por ejemplo, al conceptualizar la psicología social desde una orientación positivista, individualista y experimentalista como hizo Allport, 1954) se rastrea su historia a la luz de dicha especificación, lo cual lleva a "reescribir" el pasado (no toda la psicología social a lo largo de la historia ha sido positivista, individualista y experimentalista), sobreenfatizando la importancia de determinados desarrollos en línea con la construcción contemporánea al tiempo que otros se condenan al olvido. Si la definición que se hace de la disciplina es parcial, por mucho que provenga de posturas dominantes y claramente mayoritarias, se condena al ostracismo a una parte de la misma, y se altera la historia de la ciencia para que encaje con esta construcción moderna y parcial. En este sentido, entonces, las historias "oficiales" y legitimadoras como la de Allport tienen un efecto retrospectivo, "alterando" el pasado de la disciplina. Al mismo tiempo, al servir de base para la formación de los nuevos estudiantes de

1. Esto, a su vez, produjo un sinnúmero de anacronismos que Farr (1991) se encarga de mostrarnos. 
postgrado que se están adentrando en la disciplina, tienen un efecto prospectivo al configurar el perfil de la misma en el futuro:

Las historias oficiales se han tratado como fundamentos epistemológicos para la enseñanza de la generación siguiente para que acepte las directrices ontológicas y metodológicas de la generación actual, como se justifica y legitima por una visión selectiva de las generaciones pasadas (Lubek y Apfelbaum, 2000, p. 407, en cursiva en el original).

Hay dos consideraciones que, en este punto, merecen atención. Primero, el progresivo empobrecimiento de las sucesivas historias legitimadoras del Handbook, y segundo, su orientación presentista. En su interesante trabajo, Lubek y Apfelbaum (2000) observan cómo tales historias (primero Allport, luego Jones y luego Taylor) han ido mostrando "una progresiva degradación en la amplitud temporal, geográfica y transdisciplinar de lo que cubrían” (p. 424), de modo que:

Los subsecuentes historiadores de la psicología social del Handbook, al haber aceptado acríticamente la definición individualista de Allport como el punto de partida de sus historias, escribieron narraciones presentistas progresivamente más limitadas, dominados por su breve marco temporal personal de experiencia histórica y una falta de extensión cultural o geográfica (Lubek y Apfelbaum, 2000, pp. 423-424).

Curiosamente fue el propio Allport (1954), padre de esa limitada -y limitadora- definición a la que se refieren Lubek y Apfelbaum², quien presentó una revisión histórica que, si bien presentista y plagada de imprecisiones y errores (por ej., Haines y Vaughan, 1979; Ibáñez, 1990, pp. 12-13; Samelson, 1974, 2000), era mucho más amplia en términos temporales, reconocía los antecedentes europeos de la moderna psicología social, daba cabida a antecedentes más plurales y multidisciplinares (por ej., procedentes de la sociología) y otorgaba un papel más importante a la teorización que a la mecánica experimentación vacía de contenido que se iba instaurando progresivamente en la práctica científica de la disciplina (véase Lubek y Apfelbaum, 2000). Los capítulos de Jones $(1985,1998)$ y Taylor (1998) se centran principalmente sobre la psicología social experimental norteamericana de orientación psicológica, se limitan a fuentes mainstream, sus análisis históricos o conceptuales resultan escasos y pobres, y restan importancia a las críticas de los años 60 y 70 del pasado siglo (Farr, 1991; Lubek y Apfelbaum, 2000; Samelson, 2000). El que el capítulo histórico de Allport haya sido suprimido en la última edición del Handbook (Gilbert, Fiske y Lindzey 1998) sin ser sustituido por otro de naturaleza similar, no hace hace sino agravar el problema ${ }^{3}$.

Todo ello constituye un empobrecimiento (histórico, geográfico, epistemológico, disciplinar y conceptual) de la visión de la psicología social que se está transmitiendo a las nuevas generaciones.

2. La definición de psicología social ofrecida por Allport en las sucesivas ediciones del Handbook es la de una disciplina que intenta "entender y explicar cómo el pensamiento, el sentimiento y la conducta de las personas están bajo la influencia de la presencia real, imaginada o implicada de los demás" (Allport, 1985, p. 3). Esta fórmula (véase Lubek, 2000, nota 2 en p. 320, para algunos indicios sobre el origen de la misma) ha sido reproducida una y otra vez en un sinnúmero de libros de texto de la disciplina. Por ejemplo, Jiménez Burillo, Sangrador, Barrón y de Paúl (1992) analizaron una muestra incidental de 32 manuales de psicología social, la mitad europeos y la otra mitad norteamericanos, publicados en la década de los 80 del pasado siglo. El 68\% de los libros americanos definían la disciplina; de ellos, el 45,4\% reproducía la definición de Allport. La psicología social se definía en un 31\% de los textos europeos; en ellos también se reproducía la definición del capítulo histórico inicial del Handbook. Jiménez Burillo et al. (1992) concluyen que "de los 16 manuales de la muestra que definen la Psicología Social, el 50\% acepta la definición de G. Allport" (p. 21). La difusión de la definición de Allport refleja el impacto del Handbook of Social Psychology en la disciplina, mostrando, en línea con lo argumentado por Lubek y Apfelbaum (2000), la importancia de tales volúmenes en la formación de las nuevas generaciones de psicólogos sociales.

3. Es justo reconocer, no obstante, que algunos autores se esfuerzan en poner solución a dicho problema. Valga como muestra el número especial que, en otoño del año 2000, la revista Journal of the History of the Behavioral Sciences (vol. 36, $\mathrm{n}^{\circ} 4$ ) publicó sobre la historia de la psicología social. En la introducción, Ian Lubek (2000) indica que "esta colección contiene un abanico de perspectivas contextuales y críticas sobre el desarrollo de la psicología social -en parte como contrapunto a las historias "oficiales" o estándar, principalmente las varias ediciones del Handbook of Social Psychology..." (p. 320), y que la razón de la misma es "una postura crítica común hacia la perspectiva específica de las sucesivas representaciones historiográficas de la psicología social en los Handbooks" (p. 321, nota 4). Llama la atención la singular riqueza de las contribuciones a ese número en comparación con los limitados análisis "estándar” de Jones y de Taylor. 
Si anteriormente se ha argumentado sobre la necesidad de una conciencia histórica para entender el presente de la psicología social y su pluralidad, constatamos aquí que las sucesivas historias del Handbook van amputando progresivamente el pasado de la disciplina, al tiempo que reducen la amplitud de ésta hasta abarcar sólo la psicología social experimental. A la vista de todo ello no deben sorprendernos planteamientos como el de Berscheid (1992) al que hemos aludido anteriormente. Se está creando una psicología social limitada e históricamente huérfana.

Además de la progresiva imposición de tan estrechos límites históricos, disciplinares, epistemológicos, geográficos y conceptuales en los capítulos históricos del Handbook, una segunda consideración que es necesario formular alude a su innegable presentismo. El presentismo implica que el estado actual de la disciplina constituye su punto de desarrollo más elevado, y que se deriva natural y lógicamente de la historia previa (cual un histórico sesgo retrospectivo, Fischhoff, 1975; Hawkins y Hastie, 1990). Se establece, por ejemplo, qué es (al menos para algunos) hoy la psicología social, y desde esta perspectiva se reescribe el pasado o, como señala Ibáñez (1990), se legitima el presente buscando en el pasado la confirmación de las creencias y de los valores actuales. Como apunta ese autor, el experimentalismo de Norman Triplett y el positivismo de Auguste Comte a los que aludía Allport (1954) constituían sin duda puntos de referencia importantes para caracterizar a esa psicología social (pero no a otras). En este mismo sentido, Sabucedo et al. (1997) nos dicen que:

El trabajo de Triplett sería útil para una determinada construcción y concepción de la disciplina; permite establecer una línea de continuidad en la investigación y mostrar una acumulación de conocimientos sobre ella... Pero para poder brindar esa imagen, que resulta tan beneficiosa desde una perspectiva inductivista de la ciencia, es preciso relegar y marginar toda una serie de estudios, como los realizados sobre la sugestión, que no se adecuan al modelo de disciplina que se quiere diseñar (pp. 6-7).

Desde esta perspectiva, autores como Ibáñez (1990), Munné (1986) o Samelson (1974) cuestionan la decisión de Allport de otorgar a Comte el alto honor de la paternidad de la psicología social. Munné (1986), tras discutir los méritos de Lazarus y Steinthal, Le Bon, Tarde, Durkheim, James, Mead, Ross, McDougall, Allport, Lewin, y otros insignes antecedentes de nuestra disciplina para ser designados padres fundacionales, concluye muy acertadamente que el debate sobre la paternidad de la psicología social es pueril y estéril. Como él señala, la ciencia es un producto social, y sus diversas orientaciones surgen a través de un largo proceso emergente. Esto quiere decir que no hubo ningún momento en el cual, de pronto, apareciera la psicología social. Ésta se fue desarrollando progresivamente, tomando forma en el seno de las vecinas ciencias sociales también en desarrollo (Jiménez Burillo, 1976, 1987, la relaciona con las aportaciones hechas desde la filosofía, la sociología, la psicología y la antropología), nutriéndose de contribuciones que, en sí mismas, sería difícil ubicar en una disciplina concreta $u$ otra. Y fue desarrollándose también, sobre todo, en el seno de un marco sociohistórico determinado. La historia de la disciplina no ha sido, por añadidura, lineal, sino que ha habido vías muertas, reformulaciones y fuertes tensiones entre las diversas escuelas de pensamiento. La perspectiva lineal y triunfalista que nos ofrecen las historias presentistas como las del Handbook o la de Berscheid (1992) no constituye sino una re-construcción del pasado a partir del presente, la cual no refleja la complejidad real del desarrollo de una ciencia ni la influencia de los complejos factores múltiples, de diversa naturaleza, que, en el seno de un lewiniano "sistema de tensiones" (Ross y Nisbett, 1991), van moldeando y dando forma a las diversas modalidades de psicología social a lo largo de su devenir histórico.

Desde este punto de vista, la consideración del contexto en el que se producen los avances científicos es esencial si queremos entender el desarrollo (y, por ende, el momento actual) de la disciplina. En este sentido, Ibáñez (1990) aboga por una historia de la psicología social de carácter contextualista. Por ello entiende la necesidad de tomar en cuenta el contexto constituido por la historia de las ciencias sociales, en el sentido de que no se puede elaborar una historia separada, desgajada del devenir de las otras ciencias sociales, para la psicología social, y el contexto constituido por las condiciones sociales particulares (la revolución francesa, la industrialización, el auge militar 
e industrial de los Estados Unidos) en que se producen determinados desarrollos y que pueden, en buena medida, dar cuenta de ellos. Por ejemplo, Blanco (1993) señala como condiciones de suma importancia en el desarrollo de nuestra disciplina la rebelión contra el individualismo que aconteció en Europa hacia el siglo XVIII, el derrocamiento del antiguo régimen hacia finales del siglo XVIII, la revolución industrial y la aplicación del método científico al estudio de los problemas sociales (ver también Sabucedo et al., 1997, p. 7). Alipio Sánchez (2002) señala que:

[L]a PS [psicología social] y otras ciencias sociales son "hijas" de la modernidad ilustrada y de las turbulencias y los desarrollos sociales (revoluciones científica y política, industrialismo, capitalismo, urbanización, higiene pública, agricultura intensiva, "explosión” demográfica, etc.) e intelectuales (individualismo, secularización, utilitarismo) relacionados que tienen la fe en el progreso, el hombre y la razón como horizonte credencial unificador (p. 3).

De forma similar (y por poner algunos otros ejemplos ilustrativos):

1. Difícilmente podemos aprehender la aparición de los trabajos de Le Bon y Tarde sin ser conscientes de la gran inestabilidad social y política de la Francia de la época.

2. Tampoco podemos comprender el auge de la llamada "psicología racial" en los Estados Unidos en las primeras tres décadas del siglo XX sin saber de la inmensa oleada de inmigrantes italianos, polacos, rusos y judíos que llegaban al país y que constituían un problema social debido a su difícil integración en la sociedad norteamericana (Collier et al., 1996).

3. No se puede entender el conductismo, el individualismo y el experimentalismo de Floyd Allport (1924) como algo desgajado del contexto intelectual de la época y de la influencia de sus maestros Holt, Münsterberg y Perry en Harvard (véase Parkovnick, 2000), o como algo ajeno al "individualismo moral" (más allá del "individualismo metodológico") del propio Allport, enraizado en una concepción renacentista de la reforma y capitalista de la individualidad, muy distinta de la noción platónico-medieval de la misma (véase Greenwood, 2000).

4. Es bien conocida la influencia del nazismo sobre, por una parte, el éxodo de muchos psicólogos europeos que dieron forma a la moderna psicología social en los Estados Unidos (Cartwright, 1979), y por otra parte sobre los propios temas de investigación y reflexión psicosocial (la Psicología de Masas del Fascismo de Reich, 1933; los estilos de liderazgo de Lewin, Lippitt y White, 1939; la obediencia a la autoridad de Milgram, 1963, 1974; etc.). Asimismo, la II Guerra Mundial propició el desarrollo de un sinnúmero de estudios psicosociales (los de Lazarsfeld, 1949, sobre los soldados; los del grupo de Hovland en Yale sobre persuasión, por ej., Hovland, Lumsdaine y Sheffield, 1949; etc.) que en último término darían lugar a la madurez de la psicología social norteamericana de posguerra (por ej., Sewell, 1989).

5. Tampoco faltan quienes han vinculado la crisis de la psicología social (y las que, al mismo tiempo, sacudieron a otras disciplinas científicas) al periodo de inestabilidad sociopolítica y de crisis de los valores de finales de los 60, reflejada en las revueltas estudiantiles como el mayo francés y las manifestaciones contra la guerra del Vietnam y el auge del movimiento hippy en los Estados Unidos (Collier et al., 1996; Tolman, 1996).

6. Como último ejemplo en este punto, recordemos que no podemos entender la proliferación actual de trabajos sobre psicología del terrorismo (por ej., Echebarría Fernández, 2006; Lowenstein, 2003; Maikovich, 2005; Oswald, 2005; Ubillos, Mayordomo y Basabe, 2005; Wagner, 2006) al margen del reciente incremento del terrorismo islámico, con los acontecimientos del 11 de septiembre de 2001 en Nueva York y el 11 de mayo de 2004 en Madrid ${ }^{4}$, así como las

4. Los atentados contra el World Trade Center de Nueva York y contra el Pentágono en Washington se produjeron el 11 de septiembre de 2001. Para examinar el impacto concreto de estos acontecimientos sobre las publicaciones en psicología del terrorismo se hicieron dos búsquedas en PsychINFO. Las referencias debían contener la palabra terrorism como palabra 
invasiones estadounidenses de Afganistán e Irak, y el ya duradero problema palestino-israelí, como telón de fondo.

La influencia todavía más directa de los contextos ideológicos y políticos sobre la disciplina también ha sido puesta de manifiesto por diversos autores (por ej., Ovejero, 2000):

El darwinismo social, tal como fue desarrollado y elaborado por Herbert Spencer, enraizaba en la creencia de que son los genes superiores los que dan cuenta del éxito y del poder de las clases altas; los científicos británicos, incluidos Galton, Darwin, Pearson, Burt y Eysenck, formularon una teoría genética que apoyaba la estructura de clase. Spencer era el teórico favorito de los ilustrados norteamericanos, y William McDougall llegó a los Estados Unidos para ayudar a la psicología a dar una justificación científica para el racismo, el clasismo y la política conservadora (Albee, 1994, citado por Ovejero, 2000, p. 31).

En suma, la disciplina psicosocial (quizás, por su propia naturaleza, menos aún que otras disciplinas no-sociales) no se desarrolla al margen de sus contextos sociopolítico, histórico, económico e intelectual.

Desde este punto de vista, los textos sobre la historia disciplinar que se centran específicamente en las contribuciones individuales de grandes personajes tienen escaso valor para revelarnos los verdaderos antecedentes del devenir de una disciplina (Pepitone, 1981). En su lugar, resultan mucho más esclarecedoras ciertas aproximaciones alternativas, como la consistente en considerar el Zeitgeist de los diversos momentos históricos (es decir, "los desarrollos en otras ciencias, el clima político, el avance tecnológico y las condiciones económicas", Hergenhahn, 2000, p. 3) o la que examina cómo varias personas o hechos han ido contribuyendo a los cambios que una idea o concepto ha ido experimentando a lo largo de la historia (por ejemplo, Hergenhahn, 2000, alude al cambio que se ha producido a lo largo del tiempo sobre la idea de enfermedad mental). En definitiva, se trata de "la imperiosa necesidad de complementar el nivel de análisis meramente biográfico... con un nivel de discurso y con un nivel de análisis socioinstitucional” (Blanco, 2000, pp. 43-44).

En este sentido, Lubek y Apfelbaum (2000) abogan, como Ibáñez (1990), por aproximaciones contextualistas a la historia de la disciplina. Tales aproximaciones "implican una minuciosa inmersión en un periodo de tiempo, y no son simples cazas del tesoro de anticuarios en busca del "primer empleo del término psicología social' en un libro de texto, o el primer 'experimento' completado" (p. 421). Señalan las ventajas de este tipo de aproximación frente a una opción presentista con algunos ejemplos. En 1875 apareció en París el libro La Psychologie Sociale des Nouveaux Peuples, escrito por P. Chasles, especialista en lingüística comparada. Un historiador presentista sin duda desecharía esta obra, pese a su título, por no tener nada que ver con la actual psicología social. Pero Lubek y Apfelbaum (2000) señalan cómo desde una perspectiva contextualista, sensible a la cultura y al lenguaje, podría prestarse merecida atención al libro de Chasles, e incluso relacionarlo con las recientes perspectivas discursivas en psicología social, las cuales prestan gran atención al lenguaje (y que también son ignoradas en historias presentistas escritas desde la corriente mayoritaria).

De forma similar, Lubek y Apfelbaum (2000), al buscar los orígenes de la psicología social desde una perspectiva contextualista, prestan atención a una serie de "questions sociales" (prostitución, alcoholismo, higiene pública, etc.) que se discutían durante la segunda mitad del siglo XIX en el contexto de las entonces emergentes ciencias sociales. Como señalan los autores, tales problemas son similares a los que hoy preocupan a los miembros de la Society for the Psychological Study of

clave. La primera búsqueda se limitó al periodo comprendido entre 1998 y 2001 (4 años). Se halló que durante este período se habían publicado 42 trabajos de psicología sobre terrorismo. La segunda búsqueda se limitó al periodo de la misma duración inmediatamente posterior al 11-S, es decir, el comprendido entre 2002 y 2005 . El número de referencias contenidas en PsychINFO con terrorismo entre las palabras clave ascendió a 842. El incremento fue, por lo tanto, del $2005 \%$. 
Social Issues (SPSSI), centrados en problemas sociales y en las respuestas de la vertiente aplicada de nuestra disciplina para resolverlos. Curiosamente, las preocupaciones de los psicólogos sociales aplicados quedan al margen de los capítulos de Jones $(1985,1998)$ y Taylor (1998) del Handbook, más centrados sobre los desarrollos de la psicología social experimental (Lubek y Apfelbaum, 2000). Sin embargo, desde la psicología social aplicada se descubre un conjunto de precursores que no se encuentra desde las estrechas y presentistas posturas adoptadas por Jones y Taylor. Como dicen Lubek y Apfelbaum (2000), "esta laguna aísla sus historias de cualquier indagación de los precursores de los años 80 y 90 del siglo XVIII, indagación que los contextualistas y los psicólogos sociales de la SPSSI encontrarían reveladora" (p. 422).

En resumen:

1. Es importante tener en cuenta que el conocimiento del pasado de una disciplina es imprescindible para una adecuada comprensión del presente de la misma en toda su amplitud.

2. Sin embargo, los psicólogos sociales han mostrado poco interés por el pasado de la psicología social.

3. Esto ha ido cambiando a lo largo de las últimas décadas, pero

4. éste es un cambio limitado, ya que el desconocimiento histórico es común entre los psicólogos sociales mainstream.

5. Este desconocimiento puede deberse en buena parte al doble papel de la obligada obra de referencia en psicología social psicológica: el Handbook of Social Psychology. Este doble papel consiste en:

a. reconstruir el pasado de la disciplina, y

b. dar forma a su futuro.

6. En este sentido, resulta preocupante que los capítulos históricos del Handbook:

a. sean cada vez más restringidos en términos de la extensión del periodo histórico que abarcan (y también en su amplitud geográfica, epistemológica y metodológica, disciplinar y conceptual), así como

b. el que se escriban desde una perspectiva presentista que:

- toma el estado actual como el mejor y más desarrollado dentro de la historia de la disciplina,

- considera que el pasado ha ido conduciendo, en una línea de desarrollo lineal y ascendente, irremisiblemente a ese "glorioso" presente, y

- con ello distorsiona la historia, dejando de lado contribuciones relevantes.

7. Esta concepción presentista y lineal de la historia no refleja la realidad del desarrollo de una disciplina. Ésta típicamente presenta avances, retrocesos y líneas muertas, tensiones entre distintas escuelas de pensamiento, y está bajo la influencia del contexto histórico más amplio, del clima político, social, económico e intelectual de cada época. Estos factores sólo pueden captarse adecuadamente desde una perspectiva historiográfica contextualista.

8. En consecuencia, sólo una historia contextualista ${ }^{5}$ puede ayudarnos a entender el desarrollo de la psicología social, el porqué de sus desarrollos y orientaciones, la diversidad y complejidad del momento actual y cómo se ha llegado al mismo. Con ello se obtiene una visión más plena, rica y clarificadora de la disciplina.

5. Como las recogidas en los textos de Collier et al. (1996) o Ibáñez (1990), o en el monográfico del Journal of the History of the Behavioral Sciences (vol. $36, \mathrm{n}^{\circ} 4$ ) al que se ha aludido en la nota 3. 


\section{Referencias}

Allport, F. H. (1924). Social psychology. Boston: Houghton Mifflin.

Allport, G. W. (1954). The historical background of modern social psychology. En G. Lindzey (Ed.), The handbook of social psychology (vol. 1, pp. 3-56). Reading, MA: Addison-Wesley.

Allport, G. W. (1968). The historical background of modern social psychology. En G. Lindzey y E. Aronson (Ed.), The handbook of social psychology ( $2^{\mathrm{a}}$ ed., vol. 1, pp. 1-80). Reading, MA: Addison-Wesley.

Allport, G. W. (1985). The historical background of social psychology. En G. Lindzey y E. Aronson (Eds.), The handbook of social psychology ( $3^{\mathrm{a}}$ ed., vol. 1, pp. 1-46). Nueva York: Random House.

Bandura, A. (1990). Mechanisms of moral disengagement. En W. Reich (Ed.), Origins of terrorism: Psychologies, ideologies, theologies, states of mind (pp. 161-191). Cambridge: Cambridge University Press.

Bandura, A. (1999). Moral disengagement in the perpetration of inhumanities. Personality and Social Psychology Review, 3, 193-209.

Bandura, A. (2004). Selective exercise of moral agency. En T. A. Thorkildsen y H. J. Walberg (Eds.), Nurturing morality (pp. 37-57). Boston: Kluwer.

Berscheid, E. (1992). A glance back at a quarter century of social psychology. Journal of Personality and Social Psychology, 63, 525-533.

Blanco, A. (1993). Paternidades y filiaciones en la psicología social. Psicothema, 5(Supl.), 13-29.

Blanco, A. (2000). Aspectos históricos, epistemológicos y metodológicos de la Psicología Social en España. En A. Ovejero (Ed.), La psicología social en España al filo del año 2000: Balance y perspectivas (pp. 43-63). Madrid: Biblioteca Nueva.

Brown, R. (1965). Social psychology. Nueva York: Free Press.

Cartwright, D. (1979). Contemporary social psychology in historical perspective. Social Psychology Quarterly, 42, 82-93.

Collier, G., Minton, H. L. y Reynolds, G. (1996). Escenarios y tendencias de la psicología social. Madrid: Tecnos.

Danzinger, K. (1985). The origins of the psychological experiment as a social institution. American Psychologist, 40, 133-140.

Danzinger, K. (2000). Making social psychology experimental: A conceptual history, 1920-1970. Journal of the History of the Behavioral Sciences, 36, 329-347.

Durkheim, E. (2004). The cultural logic of collective representations. En C. Lemert (Ed.), Social theory the multicultural and classic readings (pp. 90-99). Wesleyan University: Westview Press.

Echebarría, A. y Fernández, E. (2006). Effects of terrorism on attitudes and ideological orientation. European Journal of Social Psychology, 36, 259-265.

Espinosa, P. (2001). Teorías del razonamiento sociomoral. En M. Clemente y P. Espinosa (Eds.), La mente criminal: Teorías explicativas del delito desde la Psicología jurídica (pp. 157-176). Madrid: Dykinson.

Farr, R. (1991). The long past and the short history of social psychology. European Journal of Social Psychology, 21, 371-380. 
Fischhoff, B. (1975). Hindsight/foresight: The effect of outcome knowledge on judgement under uncertainty. Journal of Experimental Psychology: Human Perception and Performance, 1, 288299.

Garrido, E., Herrero, C. y Masip, J. (2004). Teoría social cognitiva de la conducta moral y delictiva. En F. Pérez (Ed.), Serta in memoriam Alexandri Baratta (pp. 379-413). Salamanca: Ediciones Universidad de Salamanca.

Gilbert, D. T., Fiske, S. T. y Lindzey, G. (Eds.) (1998). The handbook of social psychology (4ª ed., 2 vols.). Boston: McGraw-Hill.

Graumann, C. F. (1993). Introducción a una historia de la psicología social. En M. Hewstone, W. Stroebe, J. P. Codol y G. M. Stephenson (Eds.), Introducción a la psicología social. Una perspectiva europea (pp. 21-35). Barcelona: Ariel.

Graumann, C. F. (1996). History of social psychology. En A. S. R. Manstead y M. Hewstone (Eds.), The Blackwell encyclopedia of social psychology (pp. 301-306). Oxford: Blackwell.

Greenwood, J. D. (2000). Individualism and the social in early American social psychology. Journal of the History of the Behavioral Sciences, 36, 443-455.

Haines, H. y Vaughan, G. M. (1979). Was 1898 a great date in the history of experimental social psychology? Journal of the History of the Behavioral Sciences, 15, 323-332.

Hawkins, S. A. y Hastie, R. (1990). Hindsight: Biased judgements of past events after the outcomes are known. Psychological Bulletin, 107, 311-327.

Hergenhahn, B. R. (2000). An introduction to the history of psychology (4a ed.). Belmont, CA: Wadsworth Publishing Company.

Hewstone, M. y Manstead, A. S. R. (1996). Social psychology. En A. S. R. Manstead, y M. Hewstone (Eds.), The Blackwell encyclopedia of social psychology (pp. 588-595). Oxford: Blackwell.

House, J. (1977). The three faces of social psychology. Sociometry, 40, 161-177.

Hovland, C. I., Lumsdaine, A. A. y Sheffield, F. D. (1949). Experiments on mass communication. Princeton, NJ: Princeton University Press.

Huici, C. (1987). La desindividuación en los grupos. En C. Huici (Ed.), Estructura y procesos de grupo (2a ed., pp. 31-54). Madrid: Universidad de Educación a Distancia.

Ibáñez, T. (1990). Aproximaciones a la psicología social. Barcelona: Sendai.

Íñiguez, L. (2000). Psicología Social como crítica. Emergencias de, y confrontaciones con, la Psicología Social académicamente definida en 2000. En A. Ovejero (Ed.), La psicología social en España al filo del año 2000: Balance y perspectivas (pp. 139-157). Madrid: Biblioteca Nueva.

Íñiguez, L. (2003). La psicología social como crítica: Continuismo, estabilidad y efervescencias tres décadas después de la “crisis”. Revista Interamericana de Psicología, 37, 219-235.

Jiménez Burillo, F. (1976). Psicología social en España (Notas para una historia de las ciencias sociales). Revista de Psicología General y Aplicada, 31, 235-284.

Jiménez Burillo, F. (1987). Psicología social. Madrid: Universidad de Educación a Distancia.

Jiménez Burillo, F., Sangrador, J. L., Barrón, A., y De Paúl, P. (1992). Análisis interminable: Sobre la identidad de la Psicología Social. Interacción Social, 2, 11-44.

Jones, E. E. (1985). Major developments in social psychology during the past five decades. En G. Lindzey y E. Aronson (Ed.), The handbook of social psychology (3a ed., vol. 1, pp. 47-108). Nueva York: Random House.

Jones, E. E. (1998). Major developments in five decades of social psychology. En D. T. Gilbert, S. T. 
Fiske y G. Lindzey (Eds.), The handbook of social psychology (4ª ed., vol. 1, pp. 3-57). Boston: McGraw-Hill.

Jones, E. E. y Gerard, H. B. (1967). Foundations of social psychology. Nueva York: Wiley.

Lazarsfeld, P. F. (1949). The American Soldier -an expository review. Public Opinion Quarterly, 13, 377-404.

Le Bon, G. (1895). Psychologie des foules. París: Felix Lacan.

Lewin, K., Lippitt, R. y White, R. K. (1939). Patterns of aggressive behavior in experimentally created "social climates". Journal of Social Psychology, 10, 271-299.

Lindzey, G. (Ed.) (1954). The handbook of social psychology (2 vols.). Reading, MA: AddisonWesley.

Lindzey, G. y Aronson, E. (Eds.) (1968). The handbook of social psychology (2a ed., 5 vols.). Reading, MA: Addison-Wesley.

Lindzey, G. y Aronson, E. (Eds.) (1985). The handbook of social psychology ( $3^{\mathrm{a}}$ ed., 2 vols.). Nueva York: Random House.

Lowenstein, L. F. (2003). The psychology of terrorism: Causes and remedies. En R. Roth, L. F. Lowenstein y D. R. Trent (Eds.), Catching the future: Women and men in global psychology (pp. 135-148). Lengerich: Pabst Science Publishers.

Lubek, I. (2000). Understanding and using the history of social psychology. Journal for the History of the Behavioral Sciences, 36, 319-328.

Lubek, I. y Apfelbaum, E. (2000). A critical gaze and wistful glance at Handbook histories of social psychology: Did the successive accounts by Gordon Allport and successors historiographycally succeed? Journal of the History of the Behavioral Sciences, 36, 405-428.

MacMartin, C. y Winston, A. S. (2000). The rhetoric of experimental social psychology, 1930-1960: From caution to enthusiasm. Journal for the History of the Behavioral Sciences, 36, 349-364.

Maikovich, A. K. (2005). A new understanding of terrorism using cognitive dissonance principles. Journal for the Theory of Social Behavior, 35, 373-397.

Milgram, S. (1963). Behavioral study of obedience. Journal of Abnormal and Social Psychology, 67, 371-378.

Milgram, S. (1974). Obedience to authority: An experimental view. Nueva York: Harper \& Row.

Moscovici, S. (1961). La psychanalyse, son image et son public. París: Presses Universitaires de France.

Moscovici, S. (1981). On social representations. En J. P. Forgas (Ed.), Social cognition: Perspectives on everyday understanding (pp. 181-209). Londres: Academic Press.

Munné, F. (1986). La construcción de la psicología social como ciencia teórica. Barcelona: Alamex.

Munné, F. (1991). La dominación epistemológica y la crítica externa en las ciencias sociales. Interacción Social, 1, 33-41.

Munné, F. (1993). Pluralismo teórico y comportamiento social. Psicothema, 5(Supl.), 53-64.

Munné, F. (1998). La crítica epistemológica en la Psicología Social del traspaso de siglo. En D. Páez y S. Ayestarán (Eds.), Los desarrollos de la psicología social en España (pp. 19-24). Madrid: Fundación Infancia y Aprendizaje.

Munné, F. (1999). Constructivismo, construccionismo y complejidad: La debilidad de la crítica en la psicología construccional. Revista de Psicología Social, 14, 131-144. 
Oswald, D. L. (2005). Understanding anti-Arab reactions post-9/11: The role of threats, social categories, and personal ideologies. Journal of Applied Social Psychology, 35, 1775-1799.

Ovejero, A. (2000). Necesidad de una nueva Psicología Social: Perspectivas para el siglo XXI. En A. Ovejero (Ed.), La psicología social en España al filo del año 2000: Balance y perspectivas (pp. 15-39). Madrid: Biblioteca Nueva.

Parkovnick, S. (2000). Contextualizing Floyd Allport's Social psychology. Journal of the History of the Behavioral Sciences, 36, 429-441.

Pepitone, A. (1981). Lessons from the history of social psychology. American Psychologist, 36, 972-985.

Pinillos, J. L. (2002). Postmodernismo y psicología. Una cuestión pendiente. Anales de Psicología, $18,1-11$.

Reich, W. (1933). Massenpsychologie des Faschismus. Copenhague: Verlag für Sexualpolitik.

Ross, L. y Nisbett, R. E. (1991). The person and the situation. Perspectives of social psychology. Nueva York: McGraw-Hill.

Sabucedo, J. M., D’Adamo, O. y García, V. (1997). Fundamentos de psicología social. Madrid: Siglo XXI.

Samelson, F. (1974). History, origin myth, and ideology: Comte's "discovery" of social psychology. Journal for the Theory of Social Behavior, 4, 217-231.

Samelson, F. (2000). Whig and anti-whig histories -and other curiosities of social psychology. Journal for the History of the Behavioral Sciences, 36(4), 499-506.

Sánchez, A. (2002). Psicología social aplicada. Madrid: Prentice Hall.

Secord, P. F. y Backman, C. W. (1964). Social psychology. Nueva York: McGraw-Hill.

Sewell, W. H. (1989). Some reflections on the Golden Age of interdisciplinary social psychology. Social Psychology Quarterly, 52, 88-97.

Shibutani, T. (1961). Society and personality: An interactionist approach to social psychology. Englewood Cliffs, NJ: Prentice Hall.

Stocking, G. W., Jr. (1965). On the limits of "presentism" and "historicism" in the history of the behavioral sciences. Journal of the History of the Behavioral Sciences, 1, 211-217.

Stryker, S. (1983). Tendencias teóricas de la psicología social: Hacia una psicología social interdisciplinar. En J. R. Torregrosa y B. Sarabia (Eds.), Perspectivas y contextos de la psicología social (pp. 13-72). Barcelona: Hispano Europea.

Stryker, S. (1989). The two psychologies: Additional thoughts. Social Forces, 68, 45-54.

Sykes, G. y Matza, D. (1957). Techniques of neutralization: A theory of delinquency. American Sociological Review, 22, 664-670.

Taylor, S. E. (1998). The social being in social psychology. En D. T. Gilbert, S. T. Fiske y G. Lindzey (Eds.), The handbook of social psychology ( $4^{\mathrm{a}}$ ed., vol. 1, pp. 58-95). Boston: McGraw-Hill.

Tolman, H. W. (1996). Critical social psychology. En A. S. R. Manstead y M. Hewstone (Eds.), The Blackwell encyclopedia of social psychology (pp. 144-145). Oxford: Blackwell.

Ubillos, S., Mayordomo, S. y Basabe, N. (2005). Percepción de riesgo, reacciones emocionales y el impacto del 11-M. Revista de Psicología Social, 20, 3-13.

Wagner, R. V. (2006). Terrorism: A peace psychological analysis. Journal of Social Issues, 62, 155171. 
Weigert, A. J. y Gecas, V. (1995). Multiplicity and dialogue in social psychology: An essay in metatheorizing. Journal for the Theory of Social Behavior, 25, 141-174.

Zimbardo, P. (1969). The human choice: Individuation, reason, and order versus deindividuation, impulse, and chaos. En W. J. Arnold y D. Levine (Eds.), Nebraska symposium on motivation (vol. 17, pp. 237-307). Lincoln, NE: University of Nebraska Press.

Zimbardo, P. (1997). Situaciones sociales: Su poder de transformación. Revista de Psicología Social, $12,99-112$. 